\title{
In-situ optical diagnostics of boron-doped diamond films growth
}

\author{
Maciej Kraszewski, ${ }^{*}$ Robert Bogdanowicz \\ Faculty of Electronics, Telecommunications and Informatics, Gdansk University of Technology, Narutowicza 11/12, \\ 80-233 Gdansk, Poland
}

Received October 18, 2013; accepted December 17, 2013; published December 31, 2013

\begin{abstract}
Interferometry is a desirable method for in-situ measurement of thin, dielectric film growth, as it don't modify conditions of film deposition. Here we present interferometrical measurements of thickness of doped diamond films during Chemical Vapor Deposition (CVD) process. For this purpose we used a semiconductor laser with a $405 \mathrm{~nm}$ wavelength. Additional ex-situ measurement using spectral interferometry and ellipsometry have been performed. We found that doping diamond with boron does not cause degradation of interference of light inside the film. To our knowledge, this is the first study of optical monitoring of boron doped, polycrystalline diamond films deposition.
\end{abstract}

Diamonds are valuable not only as the most expensive jewellery material, but most of all as a material, which optic, dielectric, mechanical and thermal properties [1] are not replaceable by other materials. Polycrystalline diamond films are widely used in such important fields as: microelectronics [2], electrochemistry [3] and optoelectronics [4-6].

There have been also reported attempts of coating fused silica fibres with diamond or other carbon-based materials. It is known that carbon coatings, including diamond-like carbon (DLC) can be used in biomedical applications [7]. Moreover, so called "non-active" anodes such as boron doped diamond (BDD) electrodes could be used effectively in electrochemical sensors [3,8], designed for environment monitoring and biomedical applications [9-10].

For this reason improving the Plasma Assisted Chemical Vapor Deposition (PA-CVD) process, which currently isn't controlled enough [11], is an important research problem. In-situ monitoring of film growth may be a useful tool to optimize the deposition process.

Different groups have proposed using Laser Reflectance Interferometry (LRI) with He-Ne lasers for monitoring undoped CVD diamond deposition [12-13]. However, to our knowledge, there are no published trials of applying this technique to monitoring boron doped diamond films.

LRI technique is based on measurement of film reflectance during film growth. The reflectance of a thin

\footnotetext{
*E-mail: mackrasz@pg.gda.pl
}

film with thickness $d$ and refractive index $n$, deposited on thick substrate can be modeled according to Eqs. (1)-(2):

$$
\begin{gathered}
R(d, \lambda)=\frac{R_{1}+R_{2}+2 \sqrt{R_{1} R_{2}} \cos (\beta d)}{1+R_{1} R_{2}+2 \sqrt{R_{1} R_{2}} \cos (\beta d)}, \\
\beta=\frac{4 \pi}{\lambda} n \cos \varphi,
\end{gathered}
$$

where $R_{1}$ and $R_{2}$ are the reflection coefficients from ambient-film and film-substrate respectively and $\varphi$ is the angle of incidence of laser light on the substrate (inside the film).

Diamond films used in this study have been prepared using the PA-CVD method. Monocrystalline, silicon substrate has been pre-treated by detonation nanodiamond (DND) slurry and rinsed in acetone, in order to achieve efficient diamond seeding. The dried substrate was placed inside the CVD reactor chamber. Microwave $\mathrm{H}_{2} / \mathrm{CH}_{4} / \mathrm{B}_{2} \mathrm{H}_{6}$ plasma was used for deposition. All results presented are based on diamond films prepared using plasma at pressure of 50Torr and substrate temperature of $700^{\circ} \mathrm{C}$. Samples deposited with different $\mathrm{B} / \mathrm{C}$ ration in plasma were used, in order to investigate the influence of doping on optical measurements.

Samples have been measured using three different, optical methods: in-situ measurement of reflectance of film with growing thickness - $R(d)$, ex-situ measurement of reflectance spectra of deposited films $-R(\lambda)$, and $e x$ situ spectroscopic ellipsometry.

In-situ diagnostics of diamond film growth has been performed using LRI system, depicted in Fig. 1. Instead of the classical approach with $\mathrm{He}-\mathrm{Ne}$ lasers [12-13], a semiconductor laser diode emitting light with $405 \mathrm{~nm}$ wavelength has been used. Advantages of such an approach are: better measurement resolution and interferometric contrast due to high difference in refractive indices of silicon and diamond on 405nm [1516]. The disadvantage of such a short wavelength is high absorption and scattering of light inside the film, which limits the thickness measurement range to approximately $2 \mu \mathrm{m}$ depending on a particular deposition process.

Counting the extremes of film reflectance $R(d)$ during film growth allows to calculate film thickness. Using a 
405nm light source, a measurement resolution of approximately $43 \mathrm{~nm}$ was obtained.

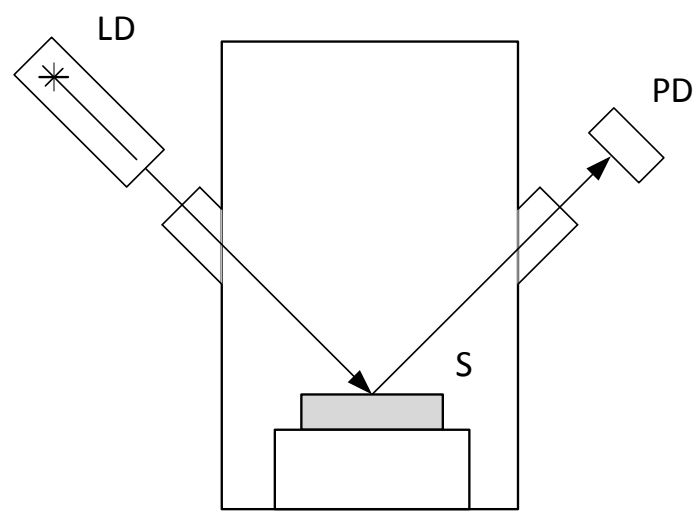

Fig. 1. Laser Reflectance Interferometry system for in-situ monitoring of CVD diamond growth. Light from laser diode (LD) is directed onto the substrate (S) inside CVD reactor chamber. Light reflected from substrate and growing thin diamond film is then measured by photodiode (PD).

Spectra of films reflectance - $R(\lambda)$ - were measured after film deposition, using a tungsten halogen light source, and normal incidence of light. This allowed to confirm the results from in-situ monitoring and to calculate film thickness with higher resolution, by fitting theoretical spectra, calculated according to Eqs. (1)-(2) to the measured ones.

To obtain better fitting of theoretical spectra, the roughness of films surface had to be considered. It was done by modifying Eqs. (1)-(2), to include the variance of film thickness, in a way presented in [17-18]. The measured and fitted theoretical spectrum $R(\lambda)$ of film with approximate thickness of $743 \mathrm{~nm}$ is presented in Fig. 2 .

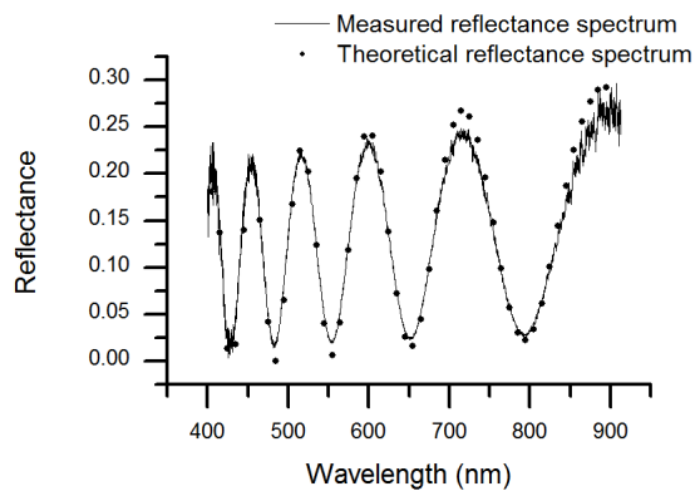

Fig. 2. Measured and theoretical reflectance $R(\lambda)$ of thin diamond film with thickness $743 \mathrm{~nm}$ and surface roughness $28 \mathrm{~nm}$.

Films were also investigated with spectroscopic ellipsometry. Ellipsometric fitting was based on a threephase optical model (air/diamond/Si-wafer). The diamond film has been assumed to be an isotropic, homogeneous material and its dispersion was fitted to the Tauc-Lorentz oscillator model. Finally, the assumed optical model was fitted to the experimental data using the non-linear Levenberg-Marquardt regression method for mean-square error minimization. As a result of SE analysis, the thickness and optical constants, i.e. refractive index $n(\lambda)$ and extinction coefficient $k(\lambda)$ were obtained.

Experimental measurement of film deposited with four different ratios of boron and carbon concentration in plasma have been performed. The 0, 2000, 5000 and 10 000B/C ratios have been chosen in order to obtain low and high film conductivity, respectively [10].

In the case of boron doped diamond films, additional attenuation of light inside the film was expected, due to the absorption caused by an electronic band structure. Such absorption should limit the measurement range of film thickness. Boron incorporation into the film has been monitored by measurement of film surface resistance, after the deposition.

The values of film reflectance $R(d)$, measured in-situ for different $\mathrm{B} / \mathrm{C}$ ratios are presented in Fig. 3. The drop of interference contrast during film growth was, in each case similar. This result suggests that the main causes of interference signal degradation are polycrystalline structure and uneven growth of the film that introduce scattering of laser light.

The accuracy of film thickness measurement with optical methods depends on the knowledge of the diamond refractive index. The value of the refractive index depends on different deposition parameters and may be difficult to predict. Table 1 presents the values of the refractive index for CVD diamond deposited with different conditions. The values have been obtained by calculating dispersion curves of each film using spectroscopic ellipsometry. The refractive index of monocrystalline diamond [16] is given as a reference.

\begin{tabular}{|c|c|}
\hline Sample & $\begin{array}{c}\text { Refractive index } \\
@ ~ 405 \mathrm{~nm}\end{array}$ \\
\hline Monocrystalline diamond [16] & 2.464 \\
\hline Undoped CVD diamond, deposited for 180 min. & 2.420 \\
\hline Undoped CVD diamond, deposited for 30 min. & 2.136 \\
\hline Boron doped CVD diamond with 5000 B/C ratio \\
in plasma.
\end{tabular}

Tab. 1. Refractive index of CVD diamond film deposited with different conditions, measured by spectroscopic ellipsometry and compared with monocrystalline diamond.

The results from Tab. 1 show that the refractive index of a CVD diamond film depends both on the dopant concentration and time of deposition and can vary within range of $10-20 \%$. We consider this main limitation of optical in-situ measurements accuracy. 


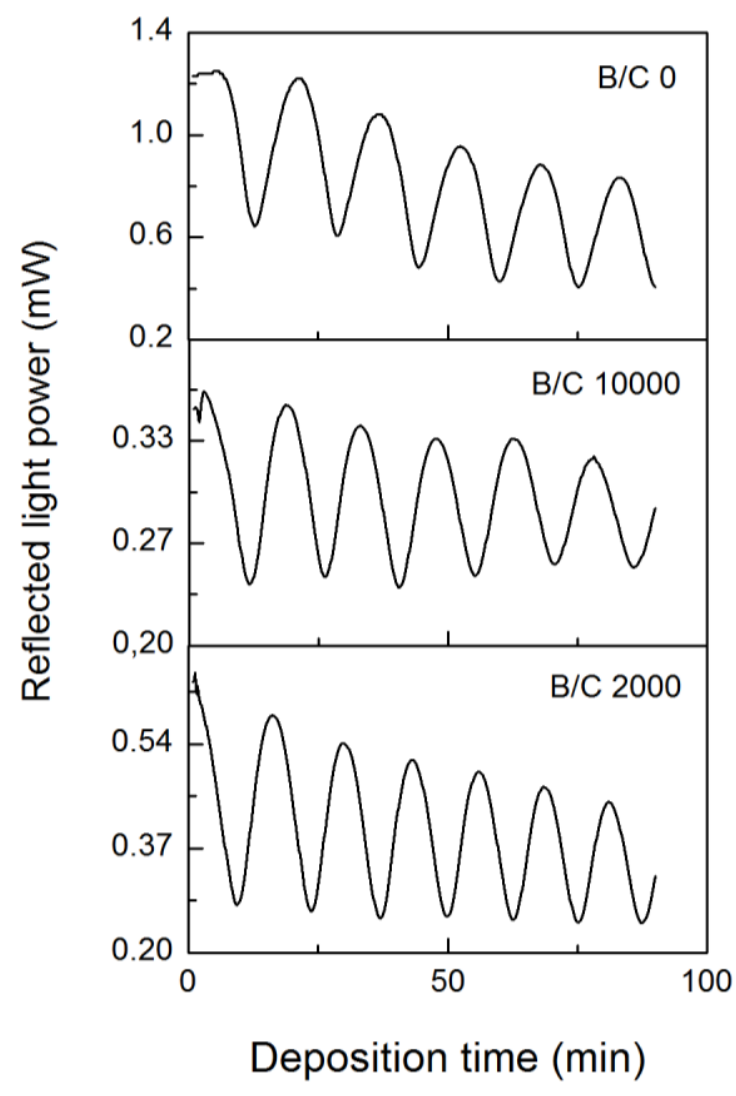

Fig. 3. Power of light reflected from thin diamond film during CVD deposition with 2000 and $10000 \mathrm{~B} / \mathrm{C}$ ratios and from undoped diamond.

Concluding, we have presented methods of noninvasive, optical diagnostics of CVD deposition of thin diamond films for electrochemical sensors. Film thickness was measured in-situ using Laser Reflectance Interferometry with a $405 \mathrm{~nm}$ semiconductor laser. We have found it to be a robust method for monitoring both dielectric, as well as semiconducting, boron doped, diamond films. However, the film refractive index may change significantly with dopant concentration, which limits the accuracy of in-situ thickness measurement.

This work was supported by the Polish National Science Center (NCN) under the Grant No. 2011/03/D/ST7/03541 and by the National Centre for Research and Development (NCBiR) under project No. LIDER/20/91/L-2/10. The DS funds of Faculty of Electronics, Telecommunications and Informatics of the Gdansk University of Technology are also acknowledged.

\section{References}

[1] J. Asmussen, D. Reinhard, Diamond Films Handbook, 1st ed. (CRC Press 2002).

[2] R.S. Sussmann, CVD diamond for electronic devices and sensors. (Chichester, U.K, J. Wiley 2009).

[3] E. Bakker,Y. Qin, Anal. Chem. 78, 12 (2006).

[4] V. Prajzler et al., Opt. Expr. 21, 7 (2013), http://www.opticsinfobase.org/oe/abstract.cfm?URI=oe-21-7-84178.

[5] N.J. Alberto et al., Proc. SPIE 8421 (2012).

[6] R. Bogdanowicz et al., Phys. Status Solidi (a) 210, 10 (2013).

[7] M. Smietana et al., Diamond Relat. Mater. 16, 4 (2007).

[8] R. Bogdanowicz et al., Sens, Actuator B-Chem. (2013); DOI: 10.1016/j.snb.2012.12.007.

[9] C.E. Nebel et al., J. R. Soc. Interface 4, 14 (2007).

[10] R. Bogdanowicz et al., Diamond Relat. Mater. 39, (2013).

[11] J.J. Gracio et al., J. Phys. D: App. Phys. 43, 37 (2010).

[12] J. Luo, X. Ying, P. Wang, L. Chen, Diamond Relat. Mater. 11, 11 (2002).

[13] A.M. Bonnot, R. Schauer, B. Weidner, Diamond Relat. Mater., 7, 2 (1998).

[14] C.D. Zuiker, D.M. Gruen, A.R. Krauss, J. Appl. Phys. 79, 7 (1996).

[15] M. Bass et al., Handbook of opitcs, Volume IV: Optical Properties of Meterials, Nonlinear Optics, Quantum Optics (McGraw-Hill 2009).

[16] E.D. Palik ed., Handbook of Optical Constants of Solids (Elsevier 1998).

[17] D.I. Siapkas, C.L. Mitsas, Appl. Opt. 34, 10 (1995).

[18] C. Katsidis, D.I. Siapkas, Appl. Opt. 41, 19 (2002). 